Document downloaded from:

http://hdl.handle.net/10251/70012

This paper must be cited as:

Alcaide Marzal, J.; Diego-Mas, JA.; Asensio Cuesta, S.; Piqueras Fiszman, B. (2013). An exploratory study on the use of digital sculpting in conceptual product design. Design Studies. 34(2):264-284. doi:10.1016/j.destud.2012.09.001.

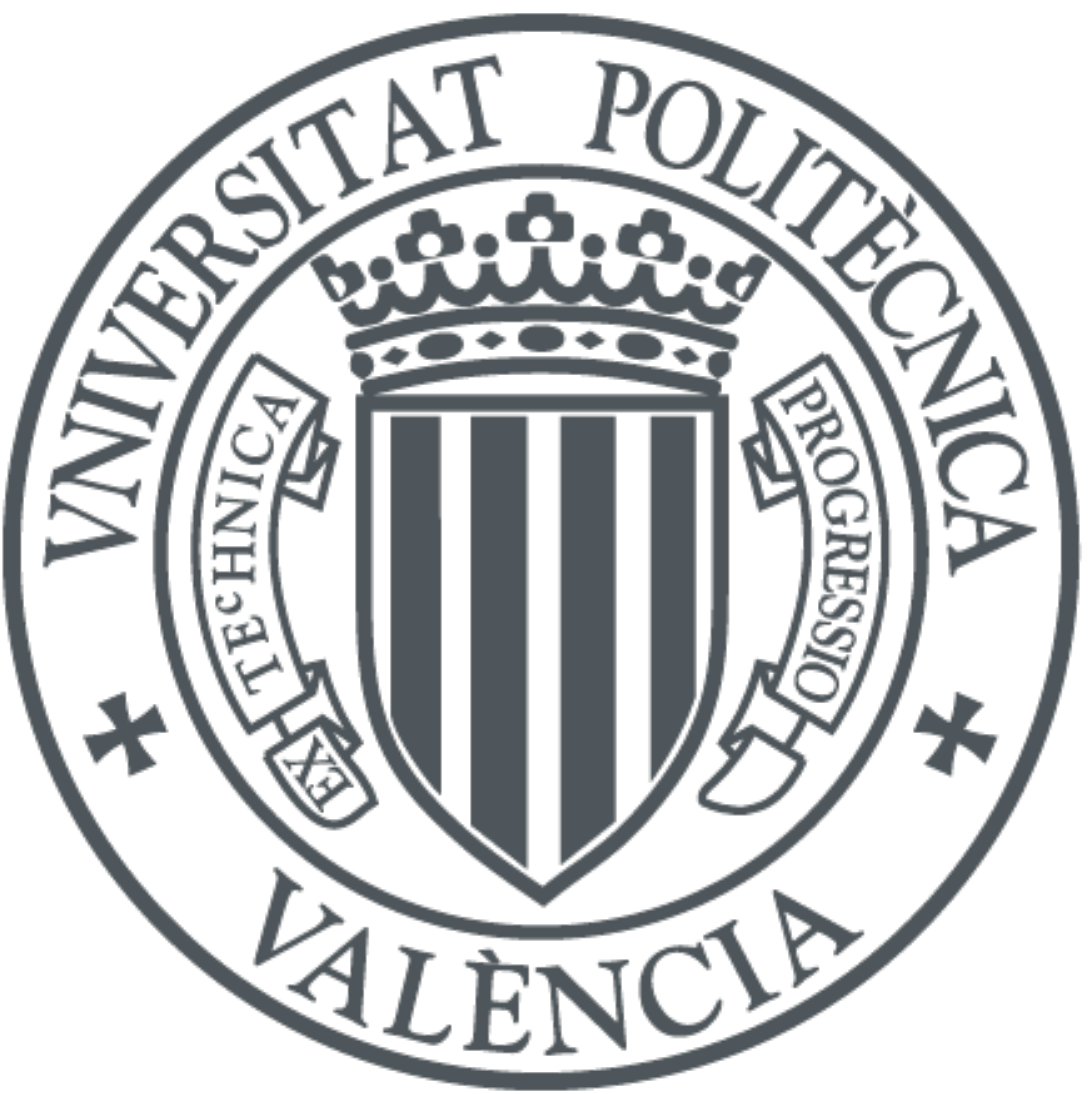

The final publication is available at

http://dx.doi.org/10.1016/j.destud.2012.09.001

Copyright Elsevier

Additional Information 


\title{
An Exploratory Study on the Use of Digital Sculpting in Conceptual Product Design
}

\begin{abstract}
The product design process involves intensive manipulation of graphical data, from pencil sketches to CAD files. The use of graphic software is common among professionals in this field. Despite this, the conceptual design stage remains intensive in paper and pencil work, as CAD systems are still too rigid to allow a creative production of concepts. In this paper the use of digital sculpting software is proposed as a way of producing 3D sketches in the early stages of the process. An experiment is conducted to determine to which extent 3D sculpt sketches can be considered as a suitable tool for conceptual design. The results show a better performance of 2D drawings, but support the complementary use of digital sculpting.
\end{abstract}

\section{Keywords}

Conceptual design; 3D Sketching; Computer aided design; Product design. 


\section{Introduction.}

Industrial product design is a problem design process which involves the intensive manipulation of graphical data, from initial sketches which roughly describe possible solutions to master CAD files useful for fabrication or analysis purposes. For this reason, the use of computers and graphic software is common among professionals in this field. But while CAD applications for detail design (modelling of parts and assemblies, inspection, preparation for manufacturing, finite element analysis, simulation, etc.) have grown exponentially, conceptual design stage is still intensive in paper and pencil work. Designers at this stage need flexibility, freshness and speed, and CAD systems, although far more flexible than their predecessors, are still too restrictive in terms of creativity. However, efforts are being made in developing 3D software applications able to meet these needs, like sketch based modelling tools and freeform modelling.

In this paper the use of digital sculpting software is proposed as an alternative to produce $3 \mathrm{D}$ sketches in the early stages of the design process. While working in a 3D environment, the tools and procedures used by this kind of software are very close to drawing practice and leave a lot of room for shape exploration, spontaneity and creativity. We argue that a freeform modelling system which uses freehand gestures and no geometric restrictions may be used so productively in the conceptual design stage as $2 \mathrm{D}$ sketching. An experiment is conducted in order to determine to which extent 3D sculpt sketches can be considered a complementary tool to $2 \mathrm{D}$ sketching for product design.

\section{Related work.}

\subsection{The role of traditional sketching in design.}

Drawings and sketches have been widely used by designers as a way to capture and develop their thoughts and ideas about a design problem (Ferguson, 1992; Cross, 2008; Pipes, 1991; Ullman, Wood, \& Craig, 1990). During the early phases of design process, when the information available is still fuzzy and abstract, designers need tools that allow them to analyze, grasp, embody and give expression to thoughts that represent partial and unfinished pieces of the object they are designing. Sketches have traditionally been one of these tools.

But sketches are more than a communication technique. As it seems nearly impossible to separate sketching and designing (Bilda, Gero, \& Purcell, 2006; McGown, Green, \& Rodgers, 1998; Purcell \& Gero, 1998; Tovey, 1989) the activity of sketching itself has been deeply analyzed by design researchers. Goldschmidt (1991) describes this activity as a dialogue 
between the designer and the sketch. Many other authors (van Sommers, 1984; Schon \& Wiggins, 1992; Goel, 1995; Suwa \& Tversky, 1997; Do, Gross, Neiman, \& Zimring, 2000) present the process of drawing sketches as an activity of conceptual design which helps designers to develop and enrich their initial ideas about the product to be designed by extracting and reusing them. Anderson \& Helstrup (1993) explain this need for sketching by resource limitations during the mental design process.

A. T. Purcell \& Gero (1998) summarize the common facts observed in the nature of sketches by several authors by providing these five characteristics: Ambiguity, reinterpretation, knowledge generation, cyclic process and expertise related. All of them are related to each other and to the mentioned capability of sketches to improve creativity.

Ambiguity is a thoroughly studied feature of sketches. They, as opposed to CAD drawings, are fuzzy, dense and unclear because designers use a particular symbolic language when sketching (Goel, 1995; Prats \& Garner, 2006; Stones \& Cassidy, 2010; Goldschmidt, 1994).

This feature is also connected to the fact that there is very little room to details when sketching (Stones \& Cassidy, 2010). Designers focus mainly in broad and general aspects of the problem, defining firstly major shapes and operators and then refining the drawings adding detail when needed. A common practice here is "overdrawing" the sketch in order to emphasize some features over others (Prats \& Garner, 2006; Cook \& Agah, 2009). Van Sommers (1984) establishes a relationship between drawing interpretation and the strokes traced during sketching.

As a consequence of ambiguity, sketches are reinterpretable. This is due to its symbolic nature, which enables new ways of seeing the same sketch. The process of reinterpretation is then activated, generating new sketches which are also reinterpretable and capable of suggesting new sketches and solutions (Stones \& Cassidy, 2010; Goldschmidt, 1994). This process is an iterative cycle which helps designers to move from abstract and conceptual knowledge to more physical one (Purcell \& Gero, 1998). The sketch then becomes a tool to generate ideas, not just a way of embodying existing ideas (Tovey, 1989; Goel, 1995; Kavakli, Scrivener, \& Ball, 1998; Company, Contero, Varley, Aleixos, \& Naya, 2009). This is regarded as one of the most valuable benefits of sketching.

According to Goel (1995), designers perform two kinds of transformations over the drawings when producing new sketches from previous ones: lateral and vertical transformations. The former are changes that produce new solutions from existing sketches, whereas the latter are refinement changes that improve and add detail to a previous sketch. This idea is also present in 
other works (Verstijnen, van Leeuwen, Goldschmidt, Hamel, \& Hennessey, 1998a) where also two kinds of possible actions, combination and restructuration, are described.

Some other works, however, conclude that sketching is not so necessary to design. (Bilda, Gero \& Purcell, 2006) shows that expert designers can produce solutions of similar quality with or without sketches. Jonson (2005) finds that the main vehicle to generate solutions in the conceptual stage is verbalization instead of freehand sketching, although other authors disagree, like Shepard (1978), cited in (Verstijnen, van Leeuwen, Goldschmidt, Hamel, \& Hennessey, 1998b). Ibrahim \& Pour Rahimian (2010) note that manual sketching tools become inadequate in highly globalised projects where many designers are involved.

Some authors have suggested that it is not sketching itself, but the externalization of thoughts, which helps designers in the production of concept ideas (Verstijnen, van Leeuwen, Goldschmidt, Hamel, \& Hennessey, 1998b). This consideration makes possible the use of other procedures of externalizing these thoughts in an equally productive way.

\subsection{Digital Sculpting and Sketches.}

The special nature of sketches has thus been an obstacle for specific CAD software to be developed in order to assist designers in conceptual design tasks. Computer tools efficiently give an answer to a wide range of design problems related to embodiment design, detailed design, simulation and manufacturing (Cross, 2008; Ullman, Wood \& Craig, 1990; Tovey, 1989; Prats, Lim, Jowers, Garner \& Chase, 2009). But in the early phases of the design process tools should be fast in producing concepts, flexible in manipulating and altering them intuitively, and unrestrictive about geometry, constraints, dimensions and so on. CAD tools are improving their performance in the conceptual stage, but still impose to the designer a very restrictive workflow when it comes to creativity and freshness (Tovey, 1989; Goel, 1995; Séquin, 2005; Lawson \& Loke, 1997; Robertson \& Radcliffe, 2009).

So the use of computers to support conceptual design is still an unresolved and developing issue (McGown, Green \& Rodgers,1998). Several studies have been conducted comparing the performance of designers using traditional and digital sketching tools. In an experiment with experienced designers, Goel (1995) found that they were more creative through traditional sketching than using constrained software, in terms of reinterpretation. Stones and Cassidy (2010) conclude in an experiment on graphic design that paper-based working is more likely to produce the reinterpretation that leads to the creation of new solutions than digital working. In a research about design strategies, they also showed that paper and pencil contributed to generate more solutions than computer tools, in all different synthesis types (Stones \& Cassidy, 2007). Bilda and Demirkan (2003) conducted an experiment about architectural design using 
traditional sketching versus digital media. Again, designers were more creative using the former, due to sketching abilities already developed and inadequacy of CAD tools. They also recommend some improvements to enhance these tools. Waburton (1996) concludes that there is no need for computer support in the conceptual phase of design. Tovey (1997) affirms that computer support should not try to replicate the sketch activity.

But other researchers hold that computers may foster creativity in this stage. For instance, Jonson (2005) found that the use of CAD could improve the generation of new concepts and patterns. Séquin (2005) showed how computer aids allow designers to explore a wider range of new shapes and design alternatives, and are even useful in producing aesthetic forms. Won, (2001) found in a study with two designers that computer demands a more complex cognitive behaviour than traditional sketching, thus supporting better reinterpretation and shifting from the whole design to details.

Madrazo, (1999) interestingly talks about the need for a dialogue between designer and machine for a true computer aided "design" to exist, in opposition to computer aided modelling. Lawson \& Loke (1997) takes this idea further, proposing a conversational CAD system.

In this sense, efforts are being made to bridge the gap between non-digital paper-based conceptual work and later (and more digitally supported) phases of the design process (McGown, Green \& Rodgers,1998). An immediate application of computers in conceptual design is the use of $2 \mathrm{D}$ drawing software to improve 2D sketching capabilities. But due to the relevance of CAD systems in the phase of embodiment and detailed design, it would be very desirable a link between 2D drawings and further 3D geometry. Thus, several approaches to this problem are being taken. An interesting review of them can be found in (Cook \& Agah, 2009) and also in (Olsen, Samavati, Sousa, \& Jorge, 2009), both describing different sketch-based modelling techniques.

Most of these techniques are based on 3D reconstruction from stroke or shading recognition (Tovey, 1997; Eggli, Hsu, Broderlin, \& Elber, 1997; Tian, Masry, \& Lipson, 2009; Company, Contero, Conesa, \& Piquer, 2004; Nealen, Igarashi, Sorkine, \& Alexa, 2007). A historical evolution of this technology can be found in (Company, Piquer, Contero, \& Naya, 2005). Van Dijk (1995) proposed a sketch based modelling system, a CAD system capable of dealing with 2D hand-sketched curves and transforming them into 3D editable volumes. Clay modelling is considered here as a promising alternative, although finally this other interesting kind of input system based in curve sketching is proposed. A reason for this is trying to mimic as much as possible the drawing gestures of $2 \mathrm{D}$ sketches. The author describes several requirements that a computer aided conceptual shape design system should fulfil which are of interest for this work. In (Verstijnen, van Leeuwen, Goldschmidt, Hamel, \& Hennessey, 1998b) a set of requirements 
for a conceptual CAD system is also proposed. Finally, some systems can be found that work directly with 3D strokes with no volume, only wireframe (Bae, Balakrishnan, \& Singh, 2008).

But our interest is focused on those techniques that rely on direct 3D meshes deformation (digital sculpting). In this kind of software, 3D sketches are produced by manipulating a basic primitive shape into complex ones representing concepts of design (Pernot, Falcidieno, Giannini, \& Leon, 2008; Cheutet et al., 2005). Manipulation is provided by different means. Although whatever PC pointing device would suffice in most cases, haptic systems (M Bordegoni \& Cugini, 2005; Sener, Pedgley, Wormald, \& Campbell, 2003; Gao \& Gibson, 2006) and virtual manipulation in augmented reality environments (Kameyama, 1997; Israel, Wiese, Mateescu, Zöllner, \& Stark, 2009; Bruno, Luchi, Muzzupappa \& Rizzuti, 2002; Bordegoni, Ferrise, Ambrogio, 2009) are found in literature. In (Séquin, 2005), a software which combines CAD capabilities with natural hands-on interaction is envisioned, in a kind of interactive digital clay modelling system.

A simpler and more similar to sketching input device is a graphic tablet. We consider that the current digital sculpting technology is developed enough to enable a real 3D sketching by mesh deformation. Nowadays, software like ZBrush, Mudbox or 3D Coat could allow designers to quickly develop 3D digital mock-ups providing almost the same flexibility, freshness and freedom that paper and pencil do, and using similar media and gestures.

The introduction of these techniques in the design process is still in a very premature stage. Some tests have been conducted (Israel et al., 2009; Kara, Shimada, \& Marmalefsky, 2007; Rahimian \& Ibrahim, 2011) to study their adequacy to designers workflow, in general presenting positive and promising conclusions in terms of externalization and concept production.

In this work we test the potential of digital sculpting with graphic tablets as a way of producing 3D digital sketches in the conceptual stage of product design. We argue that working with digital clay by using a stroke-based input device may establish a new kind of dialogue between designers and their sketches, similar to that which occurs when drawing.

\section{Material and Methods}

The aim of this work is to test the use of digital sculpting through graphic tablets as a tool for conceptual design. Our interest is to examine how designers adapt their sketching abilities to this new tool. In particular, we want to study the following hypothesis:

a) As the source of creativity is externalization, 3D sketching may provide a range of solutions similar to $2 \mathrm{D}$ sketching. 
b) Sketching skills can be assimilated to digital sculpting via graphic tablets in terms of actions and gestures.

c) Reinterpretation may occur both in 2D and 3D sketching and in similar percentages.

d) Concept construction workflows are similar in 2D and 3D sketches.

In order to explore to which extent 2D and $3 \mathrm{D}$ sketches are comparable tools in terms of concept design productivity, a comparative experiment is prepared. A group of 22 students enrolled in a subject about product conceptualization was selected to perform two sketching tasks. Students were taking a Master in Engineering Design and already had a degree in Industrial Design.

These tasks involved producing as many solutions as possible to a given design problem by means of sketching. Specifications about design were mainly aesthetical, completed with functional restrictions. Two tasks were designed for 2D and 3D sketching, separated two weeks apart (Kavakli, Scrivener \& Ball, 1998).

Participants answered several Likert-Scale questions about their use of 2D sketching and 3D software before performing these tasks. Their answers were used to separate them into groups according to their skills for further analysis.

Time given for each task was 30 minutes. This variable ranges in literature from 10-15 minutes (Prats, Lim, Jowers, Garner, \& Chase, 2009) to even weeks of observation (McGown, Green \& Rodgers,1998), depending on the purpose of the study. Given the nature of the tasks, half an hour was considered a reasonable time to produce enough conceptual solutions but preventing their refinement, as in (Stones \& Cassidy, 2010).

ZBrush 4 (Pixologic, Inc) was chosen as digital sculpting tool. Although mature in visual industries, digital sculpting techniques are still in a developing stage as conceptual design tools in many design fields. Students were trained in the use of the software with a focus on 3D sketching, but they were no experienced users. This situation was required, as a way of testing the easiness of incorporating this kind of tools into the design process.

The 2D task consisted in generating concept solutions for a new drinking fountain which was to be placed in a new museum park. Participants were provided with several photos representing the style to be achieved and some functional requirements. They were given several A4 sheets and freedom to use whatever drawing media they felt more comfortable with.

A similar task was proposed for 3D sketching. Participants were asked to design a digital stopwatch for a sport firm. Again, requirements about style and function were provided. The 
task was accomplished using ZBrush 4 and graphic tablets, and this time sketching sessions were recorded.

After both tasks, participants were asked to talk about their work in terms of number of solutions, workflow and idea generation, following the procedure described in (Jonson, 2005). This information was used to gain a better understanding of the sketching process, helping to identify the flow of solutions and reinterpretation occurrence. So research material was both quantitative and qualitative. Quantitative data was obtained from participants' sketches analysis, while qualitative information was obtained from their descriptions and from direct observation. Different indicators were used to assess the performance of individuals during the experiment:

1) Quantity of solutions: Participants were informed that they should provide as many solutions as they could. These solutions should be complete and elaborated at a conceptual level. So no detailed solutions were pursued. As aforementioned, previous works show that a lower number of solutions is expected when using digital tools.

2) Quantity of information: A major difference between 2D and 3D sketches is the amount of information generated when sketching. 2D shapes are symbolic representations (projections) of an object view onto the paper, while 3D models are virtual but complete representations of the object geometry. Designers need several 2D sketches but just one 3D sketch to completely describe an object. In order to measure the quantity of information generated by participants, a scale similar to that presented in (McGown, Green \& Rodgers,1998) was designed. A score was given to each sketch according to the following criteria:

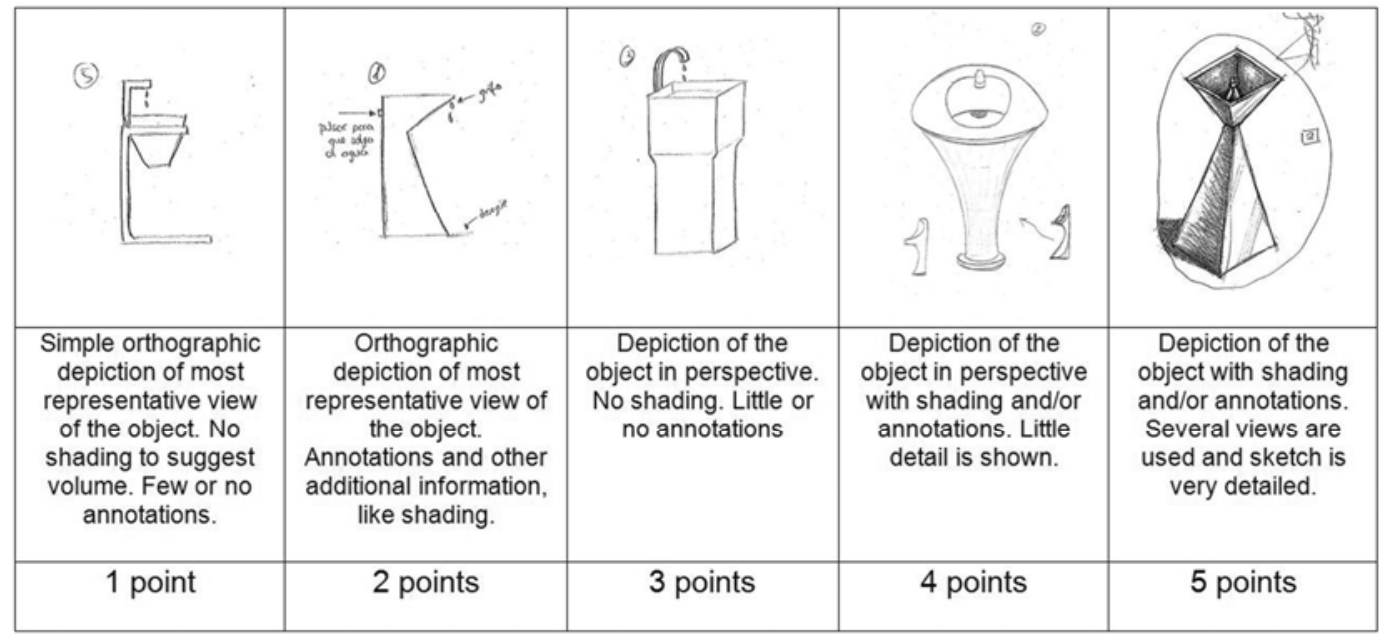

Figure 1. Sketch information scoring.

In the case of 3D sketches, obviously all of them were representations in perspective and presented in several views. No annotation was used but volume information by shading is 
automatically provided by ZBrush for any light direction. Scores varied then between 4 and 5, depending on the level of detail of the concept.

3) Number of lateral movements: Solutions were numbered and participants were asked to explain their proposals stressing the connections among them. This allowed identifying reinterpretation and the generation of new ideas out of already proposed ones.

4) Workflow and sketch transformations: A key point in this research was to test the way designers produce 3D sketches and find similarities with 2D work, if possible. The 3D task was recorded and the use of digital tools was tracked and measured in order to detect workflow patterns. Then, shape rules from the list suggested in (Prats, Lim, Jowers, Garner, \& Chase, 2009) and manipulation of predefined shapes studied in (Stones \& Cassidy, 2010) were used as a comparison basis.

\section{Results.}

\subsection{Sketch production.}

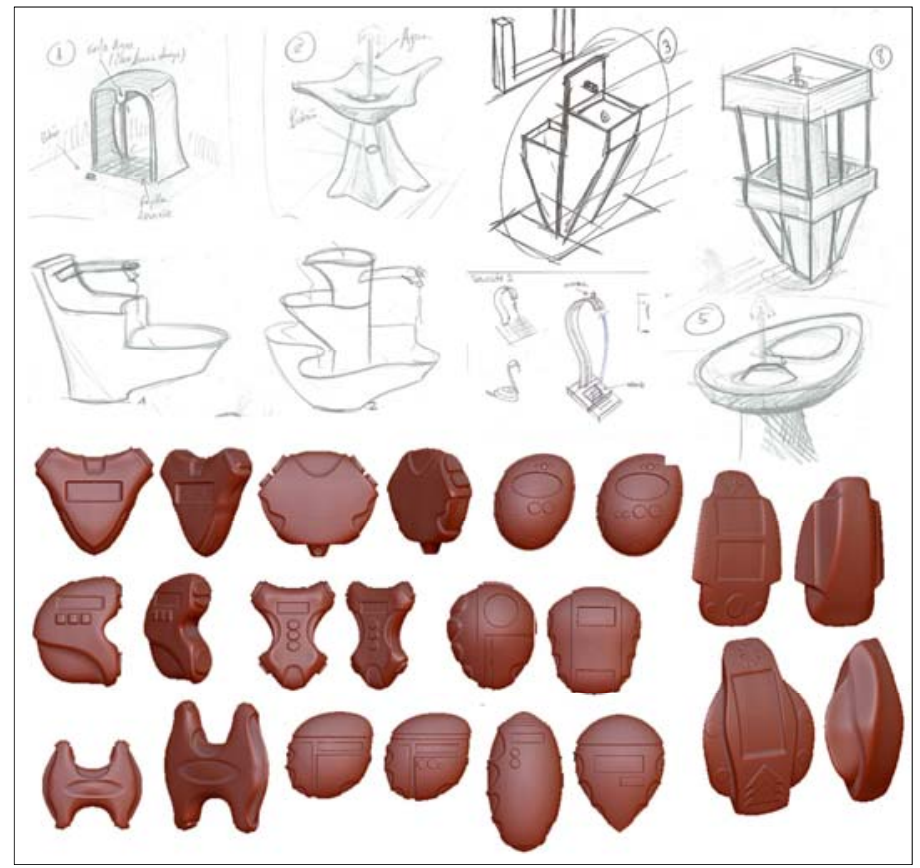

Figure 2. Examples of 2D and 3D sketches.

Participants produced 231 sketches when working with paper and pencil, coming up with 145 different solutions. 48 out of these solutions were reinterpretations derived from others. Numbers are lower when it comes to 3D sketches: 87 solutions with 19 coming from reinterpretation. The proportion of solutions coming from reinterpretation is higher in the case 
of $2 \mathrm{D}$ sketching $(33.1 \%)$ than in 3D sketching $(21.8 \%)$. The next chart displays the obtained results for both tasks.

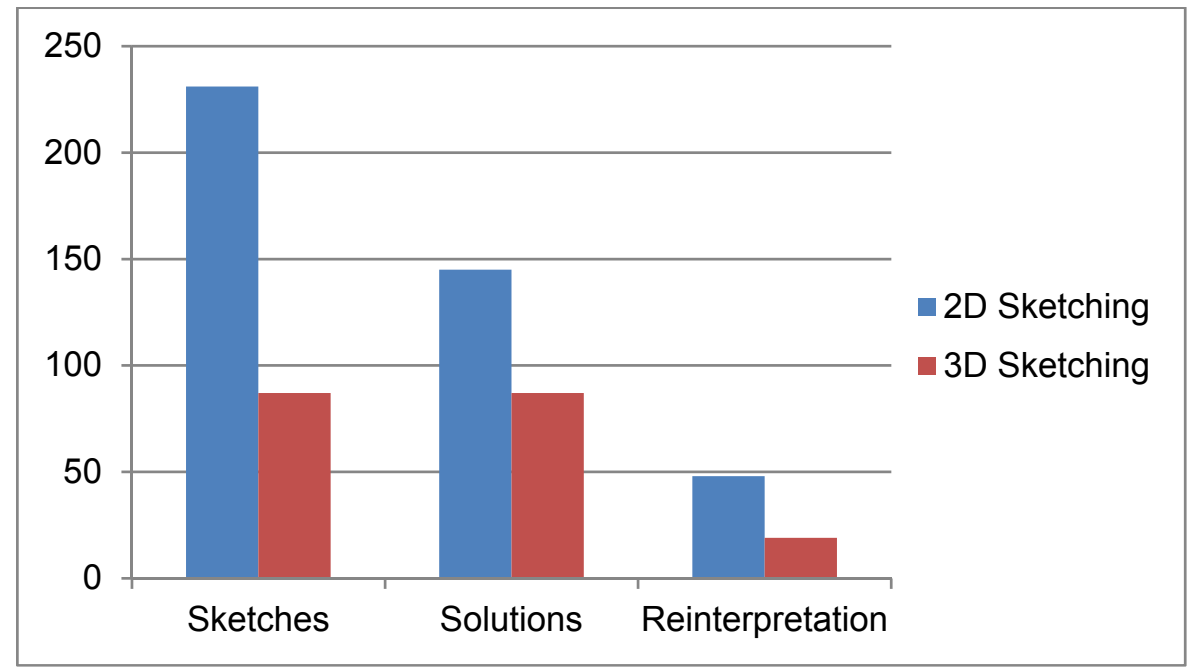

Figure 3. 2D vs 3D Sketching performance.

Each individual solution was given a score according to Figure 1 criteria, thus estimating the amount of "information" generated in each case. Only final solutions with their descriptive drawings or models were considered, as the workflow of 3D sketching does not allow capturing individual incremental "thinking" sketches: the initial base mesh evolves through sculpting to the final solution.

The amount of information generated in each task reached 430 points for $2 \mathrm{D}$ sketching and 408 for 3D sketching. This means an average of 2.96 points per solution in 2D sketches and 4.68 in $3 \mathrm{D}$ sketches. The average production per participant is summarized in the table below:

\begin{tabular}{|l|c|c|}
\hline & 2D Task & 3D Task \\
\hline Number of solutions & 6.59 & 3.95 \\
\hline Number of reinterpretations & 2.18 & 0.86 \\
\hline Quantity of information & 19.54 & 18.54 \\
\hline Time per sketch & 5.17 min. & $8.62 \mathrm{~min}$ \\
\hline Information per solution & 2.96 & 4.68 \\
\hline
\end{tabular}

Table 1. Average production per participant. 
The sketch production by group was also analyzed. Two groups were identified, according to their background and use of 2D and 3D tools in their daily work:

- $2 D$ Skilled (8 subjects): More used to sketches and illustrations, little use of design software.

- Mixers (14 subjects): They use a mix of preliminary sketches and 3D software.

The purpose of this classification was to study the relationship between participants' skills and their performance in both tasks. The average performance for each group is shown in the next chart.

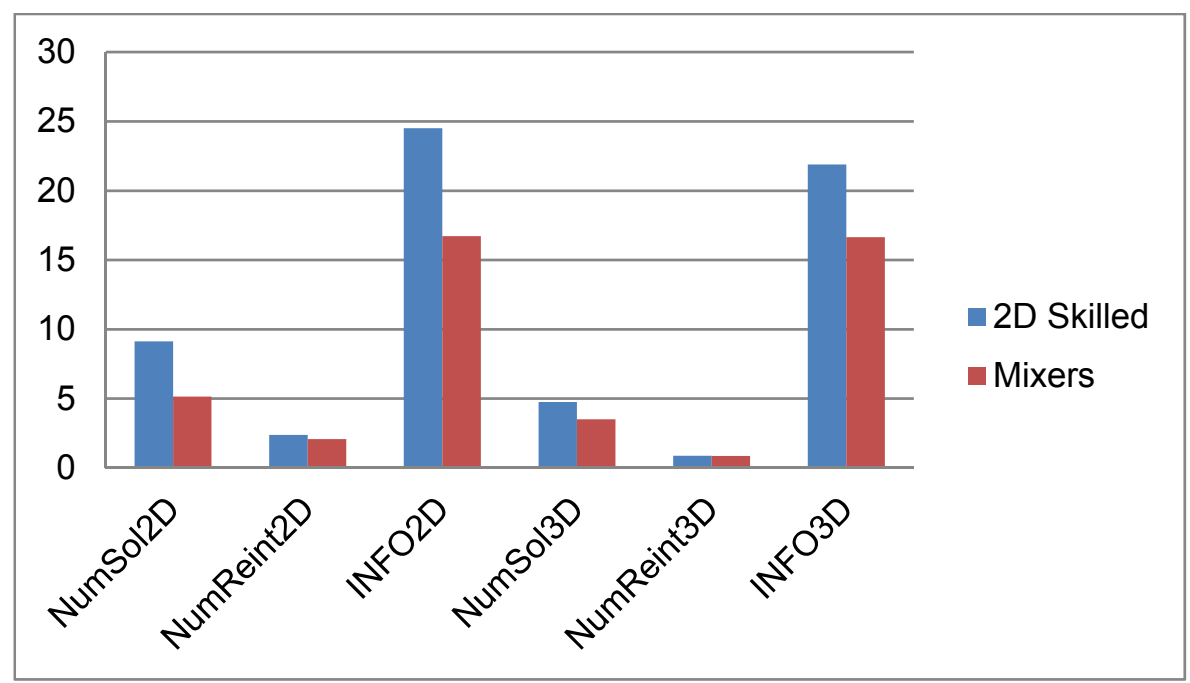

Figure 4. 2D Skilled group vs Mixer group

"Mixers" performed worst in every case in absolute terms, even in 3D tasks. Differences of performance are more evident in the number of solutions and quantity of information, whereas reinterpretation numbers are closer.

So, on the contrary, reinterpretation occurred in a higher proportion in the "Mixers" group ( $40 \%$ vs $26 \%$ in $2 \mathrm{D}$ task and $24 \%$ vs $18 \%$ in $3 \mathrm{D}$ task). They also produced a higher quantity of information per sketch ( 3.25 vs 2.68 in 2D task and 4.75 vs 4.60 in 2D task).

Although the research is mainly qualitative, a test was used to assess these differences. According to Shapiro-Wilk test two variables were not normally distributed ("Number of Solutions 3D" and "Number of Reinterpretations 3D"). Due to this and the small sample size of the experiment, a Mann-Withney test is performed in SPSS. 


\begin{tabular}{|l|r|r|r|r|r|r|}
\hline \multicolumn{7}{|c|}{ Test Statistics $^{\mathbf{b}}$} \\
\hline & N_Sol2D & N_Reint2D & INFO2D & N_Sol3D & N_Reint3D & INFO3D \\
\hline Mann-Whitney U & 19.500 & 50.500 & 20.000 & 25.500 & 56.000 & 30.500 \\
\hline Wilcoxon W & 124.500 & 155.500 & 125.000 & 130.500 & 161.000 & 135.500 \\
\hline Z & -2.501 & -.383 & -2.460 & -2.141 & .000 & -1.766 \\
\hline Asymp. Sig. (2-tailed) & .012 & .701 & .014 & .032 & 1.000 & .077 \\
\hline Exact Sig. [2*(1-tailed Sig.)] & $.010^{\mathrm{a}}$ & $.714^{\mathrm{a}}$ & $.013^{\mathrm{a}}$ & $.035^{\mathrm{a}}$ & $1.000^{\mathrm{a}}$ & $.082^{\mathrm{a}}$ \\
\hline a. Not corrected for ties. & & & & \\
\hline b. Grouping Variable: Expertise & & & & \\
\hline
\end{tabular}

Table 2. Mann-Withney Test.

Significant differences between both groups arose in number of $2 \mathrm{D}$ solutions, number of 3D solutions and 2D information. Differences in production of reinterpreted solutions were found not significant.

\subsection{D sketching workflow.}

The actions of participants were analyzed from recorded sessions and several groups of actions were identified. The aim was to explore the way concepts were generated by sculpting, establishing analogies and differences with traditional sketching workflow. Digital sculpting provides a way of modelling which strongly differs from that of CAD systems or conventional 3D software. As it makes use of freehand gestures and imposes no restrictions, the modelling context can be more easily assimilated to 2D sketching.

The use of different tools producing such actions was quantified in terms of time of use, and an average percentage of use of each tool was obtained. The different actions produced by these tools were related to $2 \mathrm{D}$ sketching actions when possible.

\begin{tabular}{|l|c|c|}
\hline \multicolumn{1}{|c|}{ Group } & Percentage & 2D equivalence \\
\hline Move & $44.86 \%$ & Outline Transformation \\
\hline Masking & $22.73 \%$ & Structure transformation \\
\hline Smooth surface & $10.81 \%$ & \\
\hline Transformation & $10.00 \%$ & Structure transformation \\
& & \\
\hline
\end{tabular}




\begin{tabular}{|l|c|c|}
\hline Add material & $5.92 \%$ & $\begin{array}{c}\text { Outline Transformation Add } \\
\text { element }\end{array}$ \\
\hline Predefined shapes & $3.86 \%$ & - \\
\hline Cut & $1.82 \%$ & Cut element \\
\hline
\end{tabular}

Table 3. 3D actions analysis.

The most performed action was "Move". It produces a deformation on the mesh, locally changing its shape. In the designer's workflow, this is equivalent to the outline transformation in 2D. When sculpting, the designer tries to find the shape by deforming the mesh and getting feedback. There is no "overdrawing" because there is no "drawing" at all, but the effect is a transition through all possible stages from the original shape to the final one. An example of this workflow is shown in the attached video.

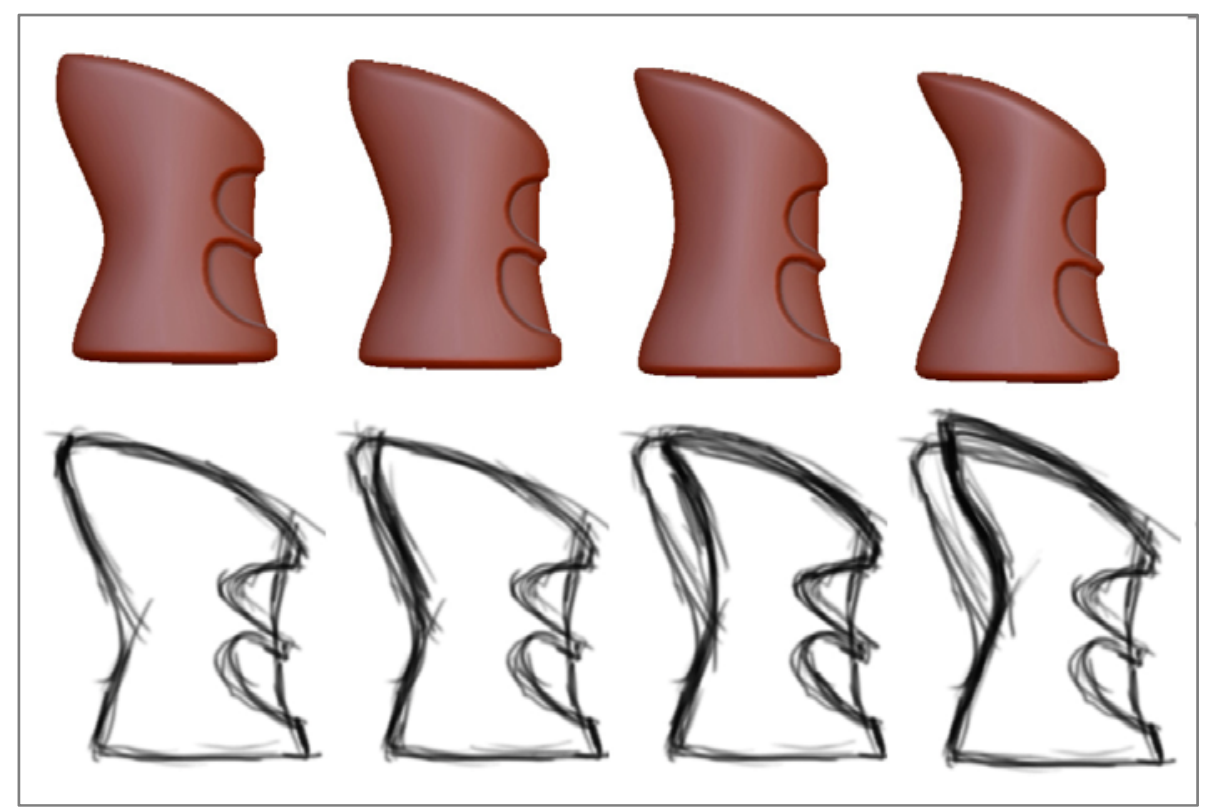

Figure 5. Analogy between "Move" action and overdrawing.

"Masking" is a tool often included in sculpting software. Its purpose is to preserve some areas of the mesh surface from the action of other tools. It is very common in $2 \mathrm{D}$ graphic design software. In 3D sculpting it is usually used to apply a homogeneous effect over the unmasked area. In most observed cases, this effect was a displacement or scaling of the area, so it has been linked to "Structure transformation". 
"Smoothing" is a very common action when sculpting, aimed to level off the surface after another action. No equivalent 2D transformation has been found. It could be similar to erasing auxiliary lines after overdrawing, leaving just the main one.

The action "Transformation" refers to displacement, rotation or scale variation of the whole mesh or one part. It differs from "Move" in the way the displacement is produced (the whole affected area receives the same variation). So it has been related to "Structure transformation".

The action "Add material" involves the use of tools whose effect is the addition or substraction of volume by freehand strokes. When the addition is slight and usually used in combination with Smooth, this action can be related to Outlining, as the result is like adding a new line and refining in 2D. If the effect is strong, then it is more similar to "Add element" or "transformation. Most of the time it was used the first way.

The use of predefined shapes is not considered in (Prats, Lim, Jowers, Garner, \& Chase, 2009) but is analyzed in (Stones \& Cassidy, 2010) and as the use of ready-made shapes is common in digital sculpting, it was measured here. This is an action which generally produces a protrusion in the surface of the mesh with the form of a predefined shape. Its use was relatively low, indicating that participants preferred other ways of detailing their concepts.

Finally, the action "Cut" evenly removes a part of the mesh. This directly relates to the Cut Transformation, but participants used this tool only to remove little parts of the mesh or to generate planar aesthetic surfaces.

The use of these tools by the two groups was also studied, trying to detect different patterns of use depending of each group expertise. The results are displayed in the next chart, in terms of relative usage time.

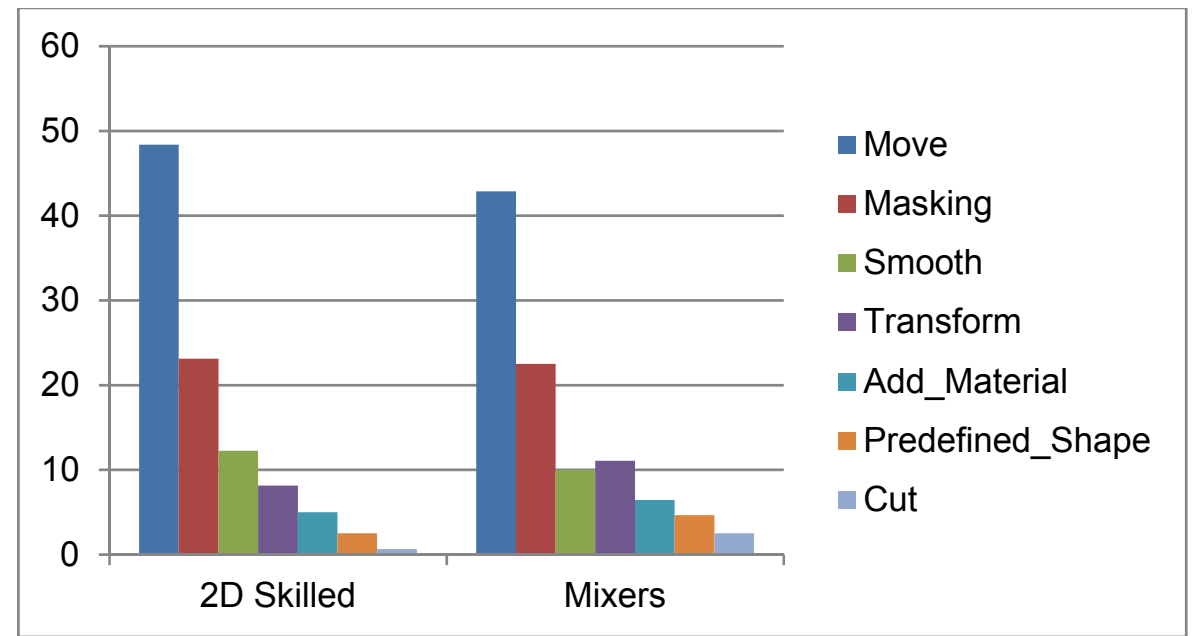

Figure 6. Comparing 3D action patterns. 
Patterns are very similar. Both groups show an intense use of "Move" and "Masking" actions and different frequencies of use of the other tools. "2D Skilled" group makes a greater use of "Move" and a very low use of predefined shapes or cutting actions. As expected, MannWithney test performed over these groups shows no significant difference in any variable.

\section{Conclusions.}

The results of the study agree in general with those of previous works comparing traditional and digital tools. Paper and pencil are still more powerful than digital media to produce conceptual solutions, in terms of number of concepts. This evidence rejects our first hypothesis, as participants were more productive when using 2D sketches. With respect to lateral movements, our third hypothesis is partially supported. The percentage of solutions coming from reinterpretation in 3D task $(21.8 \%)$ is high and close to $2 \mathrm{D}$ task one. This shows that reinterpretation may also occur frequently while sculpting. Even so, the proportion of lateral solutions was higher in $2 \mathrm{D}$ task.

Interestingly, individuals who were more used to $2 \mathrm{D}$ sketching performed better also in the $3 \mathrm{D}$ task. This result supports the second hypothesis. In previous works comparing traditional and digital tools, the latter have always been either vector based 2D graphics software (Illustrator, Freehand) or CAD packages (Pro-Engineer, Design Apprentice). As aforementioned, digital sculpting is quite different from traditional modelling. Although spatial vision and other visual abilities are likewise required, tools and procedures are closer to artistic production than to CAD methods. A graphic tablet with pressure levels is used as input device and most of the operations are produced by freehand gestures. Digital sculpting is closer to traditional drawing and sketching and so CAD skills are of little value, while drawing skills have proved being more helpful.

The amount of information produced in $2 \mathrm{D}$ and $3 \mathrm{D}$ tasks is similar. Although the way of measuring this information is merely an approximation, it was observed during the experiments and from the reports that participants were more inclined to detailing in 3D sketches. They also felt a need for sculpting completely each mesh, even when some parts of the product were not going to be visible. In fact, all the concepts produced during the experiment were fully sculpted.

Thus, average time per sketch was higher in 3D tasks. This was an expected result, as participants were not expert users of the software. The aim was to observe differences in workflows. A higher average time per sketch does not necessarily mean that people were less productive when 3D sketching, as part of this extra time was devoted to further develop their concepts. It could be objected that this constitutes an obstacle in a conceptual stage, when a lot 
of concepts are required and detailing should not be a priority. It may be true, but we think the problem is not the tool itself, but the way it is used.

Our perception is that people using 3D software feel a need for completely defining the designed shape. The possibility of 3D navigation seems to compel the designer to work on every face of the concept. In (Bilda \& Demirkan, 2003) this change in the workflow due to the difference between traditional and digital tools capabilities was also documented. Besides, the computer conveys the designer a sense of being in a more advanced stage, as if $3 \mathrm{D}$ sketches had to be more elaborated than simple conceptual drawings.

It is also worth noting that even participants skilled in traditional sketching altered their workflow when producing 3D concepts. They generated fewer solutions, and invested more time working on each mesh than on each drawing. And they also spent more time detailing their 3D concepts and fully sculpting them than when drawing, even when they were used to work with conceptual sketches. This agrees with previous studies (Madrazo, 1999; Stones \& Cassidy, 2010) which hold that the kind of tool used to produce conceptual solutions influences the way they are produced.

Despite this, the analysis of 3D actions showed an interesting resemblance between 2D and 3D sketching workflow. Paper and pencil are still more flexible tools and allow reinterpretation to occur more easily, as provide more ambiguous shapes and a faster way of redirecting the design flow. But playing with digital clay and letting forms emerge is a very similar task. Five out of the seven shape transformations listed in (Prats, Lim, Jowers, Garner, \& Chase, 2009) were used by participants while sculpting (including "Change of View", something that is almost constant in 3D modelling). The most frequent action when 3D sketching was "Move": Participants begin to deform an initial shape looking for appealing concepts, aiming at a main structure but playing with differences in the contour as seen from various points of view, shrinking and stretching areas just getting the feeling. The way of performing this is very close to the "overdrawing" practice in 2D. In some sense it could be said that participants used "3D overdrawing" most of the time. Masking was also mostly used by freehand, drawing mask areas to perform a transformation over them later on.

Obviously, 2D and 3D approaches present strong differences. 3D sketching needs an initial shape, which may influence the direction of the solutions. The designer works most of the time on a clean form, which may hamper the ambiguity needed for reinterpretation. 3D sculpting allows shape inspection from any point of view, thus compelling the designer to complete the concept even if there is no need to do so. 
But all these differences, intrinsic to the nature of 3D graphic systems, are not an actual obstacle to the use of digital sculpting as a productive design tool. 3D sketching has proved to produce reinterpretation anyway, and a reasonable range of well detailed solutions. Work system developed by participants when sculpting presents similarities with 2D drawing and provides room for free exploration and creativity. People skilled in drawing have made a more efficient use of digital sculpting.

The influence of traditional CAD use is reflected in the behaviour of participants using computers. Several works (Stones \& Cassidy, 2010; Company, Contero, Varley, Aleixos \& Naya, 2009; Madrazo, 1999) emphasize the importance of distinguishing between "designing" (creation of solutions) and modelling (production of shapes) when teaching computer design. The nature of traditional CAD systems usually imposes its workflow routine over the designer creative flow, as CAD is usually used for detail design and manufacturing. During this experiment, participants felt more free and unrestricted when drawing, and so they worked more "conceptually". The use of computers, even when the software allowed a pure conceptual workflow, made them work as if the model were not a concept but a definitive product.

The conclusion is that computer tools for conceptual design are already available, but it is difficult to use them properly. A new approach to computer aided design is needed in order to take advantage of them. The dialogue between designer and digital sculpting is actually there, but it needs some adjustments.

It also seems that the feeling of traditional sketching is still very difficult to replace. We suggest instead that blending digital sculpting with further sketching over the mesh surface or using digital clay as a second concept generation stage after very rough sketches could produce a more effective workflow.

One of the limitations of this study is sample size. Other similar works have been reported with low sample sizes: ten in (Stones \& Cassidy, 2010) and (Jonson, 2005), six in (Bilda \& Demirkan, 2003), two in (Won, 2001). The nature of these experiments and the analysis of collected data usually require a limited sample size, which is compensated by the richness of the output. The other main limitation is in our opinion the unavoidable artificial nature of the experiment. Future works should try to compare not the results, but the "experience" of drawing against that of sculpting. Another interesting approach would be comparing different methods of producing 3D sketches, providing a more in depth analysis of freeform sculpting by designers. The aim is, in short, to identify the keys of this new kind of interaction between designers and computers. 


\section{References}

Anderson, R. E., \& Helstrup, T. (1993). Visual discovery in mind and on paper. Memory cognition, 21, 3; 283-293.

Bae, S.-H., Balakrishnan, R., \& Singh, K. (2008). ILoveSketch: as-natural-as-possible sketching system for creating 3D curve models. In S. B. Cousins \& M. BeaudouinLafon (Eds.; Proceedings of the 21st annual ACM symposium on User interface software and technology (pp. 151-160). ACM.

Bilda, Z., \& Demirkan, H. (2003). An insight on designers' sketching activities in traditional versus digital media. Design Studies, 24, 1; 27-50.

Bilda, Z., Gero, J. S., \& Purcell, T. (2006). To sketch or not to sketch? That is the question. Design Studies, 27, 5; 587-613.

Bordegoni, M, \& Cugini, U. (2005). Haptic modelling in the conceptual phases of product design. Virtual Reality, 9, 2-3; 192-202.

Bordegoni, M., Ferrise, F., Ambrogio, M., (2009). Haptic Interaction and Interactive Simulation in an AR Environment for Aesthetic Product Design. Proceedings of the 3rd International Conference on Virtual and Mixed Reality: Held as Part of HCI, 293-302.

Bruno, F., Luchi, M., Muzzupappa, M., \& Rizzuti, S. (2002). A Virtual Reality desktop configuration for free-form surface sketching. XIV INGEGRAF, Santander, Spain.

Cheutet, V., Catalano, C., Pernot, J., Falcidieno, B., Giannini, F., \& Leon, J. (2005). 3D sketching for aesthetic design using fully free-form deformation features. Computers \& Graphics, 29, 6; 916-930. 
Company, P, Piquer, A., Contero, M., \& Naya, F. (2005). A survey on geometrical reconstruction as a core technology to sketch-based modelling. Computers \& Graphics, 29, 6; 892-904.

Company, P., Contero, M., Conesa, J., \& Piquer, A. (2004). An optimisation-based reconstruction engine for 3D modelling by sketching. Computers \& Graphics, 28, 6; 955-979.

Company, P., Contero, M., Varley, P., Aleixos, N., \& Naya, F. (2009). Computer-aided sketching as a tool to promote innovation in the new product development process. Computers in Industry, 60, 8; 592-603.

Cook, M. T., \& Agah, A. (2009). A survey of sketch-based 3-D modelling techniques. Interacting with Computers, 21, 3; 201-211.

Cross, N. (2008). Engineering Design Methods: Strategies for Product Design. Wiley.

Do, E. Y.-L., Gross, M. D., Neiman, B., \& Zimring, C. (2000). Intentions in and relations among design drawings. Design Studies, 21, 5; 483-503.

Eggli, L., Hsu, C. yao, Bruderlin, B. D., \& Elber, G. (1997). Inferring 3D models from freehand sketches and constraints. Computer-Aided Design, 29, 2; 101-112.

Ferguson, E. S. (1992). Engineering and the Mind's Eye. MIT Press.

Gao, Z., \& Gibson, I. (2006). Haptic sculpting of multi-resolution B-spline surfaces with shaped tools. Computer-Aided Design, 38, 6; 661-676.

Goel, V. (1995). Sketches of Thought. MIT Press.

Goldschmidt, G. (1994). On visual design thinking: the vis kids of architecture. Design Studies, 15,$2 ; 158-174$.

Goldschmidt, Gabriela. (1991). The dialectics of sketching. Creativity Research Journal, 4, 2; 123-143.

Ibrahim, R., \& Pour Rahimian, F. (2010). Comparison of CAD and manual sketching tools for teaching architectural design. Automation in Construction, 19, 8; 978-987. 
Israel, J. H., Wiese, E., Mateescu, M., Zöllner, C., \& Stark, R. (2009). Investigating three-dimensional sketching for early conceptual design-Results from expert discussions and user studies. Computers \& Graphics, 33, 4; 462-473.

Jonson, B. (2005). Design ideation: the conceptual sketch in the digital age. Design Studies, 26, 6; 613-624.

Kameyama, K.-ichi. (1997). Virtual Clay Modelling System. Proceedings of the ACM symposium on Virtual reality software and technology VRST 97, 197-200.

Kara, L. B., Shimada, K., \& Marmalefsky, S. D. (2007). An evaluation of user experience with a sketch-based 3D modelling system. Computers \& Graphics, 31, $4 ; 580-597$.

Kavakli, M., Scrivener, S. A. R., \& Ball, L. J. (1998). Structure in idea sketching behaviour. Design Studies, 19, 4; 485-517.

Lawson, B., \& Loke, S. M. (1997). Computers, words and pictures. Design Studies, 18, 2; 171-183.

Madrazo, L. (1999). Types and Instances: a paradigm for teaching design with computers. Design Studies, 20, 4; 177-193.

McGown, A., Green, G., \& Rodgers, P. A. (1998). Visible ideas: information patterns of conceptual sketch activity. Design Studies, 19, 4; 431-453.

Nealen, A., Igarashi, T., Sorkine, O., \& Alexa, M. (2007). FiberMesh : Designing Freeform Surfaces with 3D Curves. ACM Transactions on Graphics TOG, 26, 3; 41.

Olsen, L., Samavati, F. F., Sousa, M. C., \& Jorge, J. A. (2009). Sketch-based modelling: A survey. Computers \& Graphics, 33, 1; 85-103.

Pernot, J, Falcidieno, B., Giannini, F., \& Leon, J. (2008). Incorporating free-form features in aesthetic and engineering product design: State-of-the-art report. Computers in Industry, 59, 6; 626-637. 
Pipes, A. (1991). Drawing for 3-Dimensional Design. Brown \& Benchmark Pub.

Prats, M., \& Garner, S. (2006). Observations on ambiguity in design sketches. TRACEY Online Journal of Contemporary Drawing Research.

Prats, M., Lim, S., Jowers, I., Garner, S. W., \& Chase, S. (2009). Transforming shape in design: observations from studies of sketching. Design Studies, 30, 5; 503-520.

Purcell, A. T., \& Gero, J. S. (1998). Drawings and the design process: A review of protocol studies in design and other disciplines and related research in cognitive psychology. Design Studies, 19, 4; 389-430.

Rahimian, F. P., \& Ibrahim, R. (2011). Impacts of VR 3D sketching on novice designers' spatial cognition in collaborative conceptual architectural design. Design Studies, 32, 3; 255-291.

Robertson, B. F., \& Radcliffe, D. F. (2009). Impact of CAD tools on creative problem solving in engineering design. Computer-Aided Design, 41, 3; 136-146.

Schon, D. A., \& Wiggins, G. (1992). Kinds of seeing and their functions in designing. Design Studies, 13, 2; 135-156.

Sener, B., Pedgley, O., Wormald, P., \& Campbell, I. (2003). Incorporating the FreeForm Haptic Modelling System into New Product Development. Proceeding of EuroHaptics 2003, p. 26-38.

Shepard, R. N. (1978). Externalization of mental images and the act of creation. In B. S. Randhawa \& W. E. Coffman (Eds.); Visual Learning Thinking and Communication (pp. 133-189). Academic Press.

Stones, C., \& Cassidy, T. (2007). Comparing synthesis strategies of novice graphic designers using digital and traditional design tools. Design Studies, 28,1; 59-72.

Stones, C., \& Cassidy, T. (2010). Seeing and discovering: how do student designers reinterpret sketches and digital marks during graphic design ideation? Design Studies, 31, 5; 439-460. 
Suwa, M., \& Tversky, B. (1997). What do architects and students perceive in their design sketches? A protocol analysis. Design Studies, 18, 4; 385-403.

Séquin, C. H. (2005). CAD tools for aesthetic engineering. Computer-Aided Design, 37, $7 ; 737-750$.

Tian, C., Masry, M., \& Lipson, H. (2009). Physical sketching: Reconstruction and analysis of 3D objects from freehand sketches. Computer-Aided Design, 41, 3; 147-158.

Tovey, M. (1989). Drawing and CAD in industrial design. Design Studies, 10, 1; 24-39.

Tovey, M. (1997). Styling and design: intuition and analysis in industrial design. Design Studies, 18, 1; 5-31.

Ullman, D. G., Wood, S., \& Craig, D. (1990). The importance of drawing in the mechanical design process. Computers \& Graphics, 14, 2; 263-274.

Verstijnen, I. M., van Leeuwen, C., Goldschmidt, G., Hamel, R., \& Hennessey, J. M. (1998a). Creative discovery in imagery and perception: Combining is relatively easy, restructuring takes a sketch. Acta Psychologica, 99, 2; 177-200.

Verstijnen, I. M., van Leeuwen, C., Goldschmidt, G., Hamel, R., \& Hennessey, J. M. (1998b). Sketching and creative discovery. Design Studies, 19, 4; 519-546.

Warburton, N. (1996). A heuristic model for Digitally Integrated Design. Co-Design, (Double issue) $7 \& 8 ; 22-27$.

Won, P.-H. (2001). The comparison between visual thinking using computer and conventional media in the concept generation stages of design. Automation in Construction, 10, 3; 319-325.

Van Dijk, C. G. C. (1995). New insights in computer-aided conceptual design. Design Studies, 16,$1 ; 62-80$.

Van Sommers, P. (1984). Drawing and cognition: Descriptive and experimental studies of graphic production processes. Cambridge University Press . 